\title{
NEW RESULTS FROM COMPLETE SAMPLES OF FAINT RADIO GALAXIES AND QUASARS
}

\author{
K.M. BLUNDELL ${ }^{1}$, S. RAWLINGS ${ }^{1}$, S.A. EALES ${ }^{2}$, M. LACY $^{1}$ \\ 1 Oxford University Astrophysics - Keble Road, Oxford, \\ OX1 3RH, U.K. \\ ${ }^{2}$ Department of Physics \& Astronomy, University of Wales \\ at Cardiff, CF2 3YB, Wales, U.K.
}

1. Improved coverage of the luminosity-redshift plane
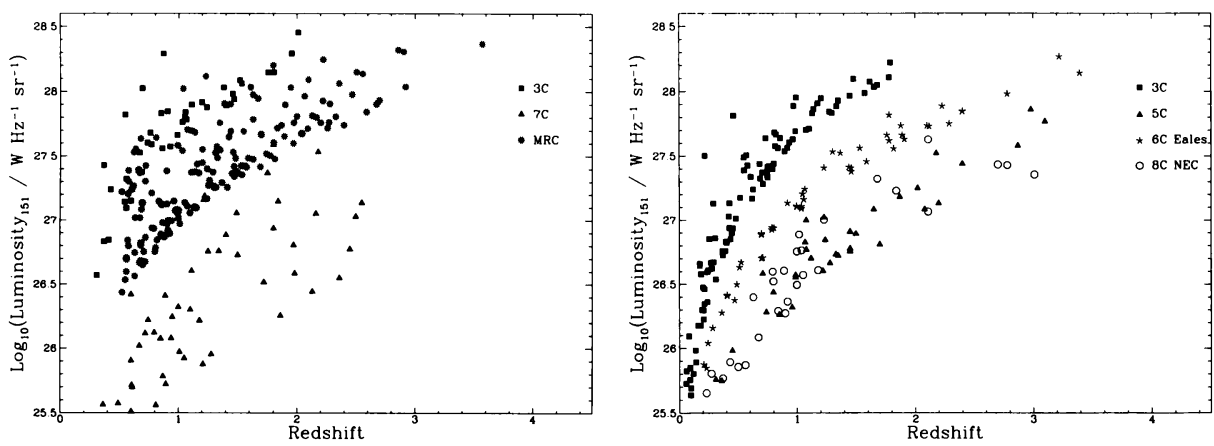

Figure 1. Coverage of the $P-z$ plane with the new flux-limited quasar (left) and RG (right) samples overlaid on the coverage from $3 \mathrm{C}$.

In any flux-limited sample a tight correlation of luminosity $(P)$ and redshift $(z)$ is inevitable. It is therefore necessary to obtain complete samples at lower and lower flux-limits in order to have adequate coverage of the $P-z$ plane, essential if we are to decouple the trends in epoch from trends in luminosity. This we have done for a number of flux-limits - giving coverage of the $P-z$ plane seen in Fig. 1. Our redshift information is spectroscopic; the results of Eales et al (in prep.), namely the increased scatter in the 

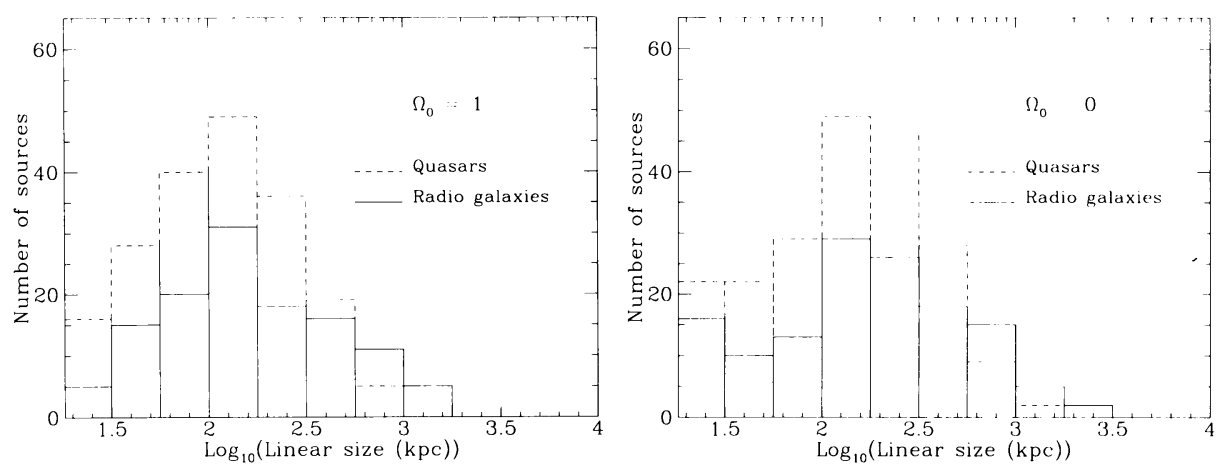

Figure 2. Histograms of the linear sizes of RGs and quasars calculated for two values of $\Omega$.

$K-z$ plot for samples lower in luminosity than $3 \mathrm{C}$, strongly warn us against using redshifts estimated from $K$-magnitudes.

\section{Linear size evolution of radio sources}

We calculated the three-way partial rank correlation coefficients (Macklin 1982) for the linear sizes $(D)$ of sources, with their redshifts and luminosities. For a universe with $\Omega=1$, we obtain for both radio galaxies and quasars in our complete samples, $r_{D z \mid P}=-0.43$ with significance $7.6 \sigma$ and $r_{D P \mid z}=-0.0067$ with significance $0.11 \sigma$. (The notation $r_{D z \mid P}$ means the partial rank correlation coefficient between $D$ and $z$ at constant $P$ ). We thus find a strong anti-correlation between $D$ and $z$, but not between $D$ and $P$.

\section{Comparison of the linear sizes of radio galaxies and quasars}

Barthel (1989) found the median linear size of RGs in 3C to be $\sim 2.2$ times that of the quasars in $3 \mathrm{C}$, lending strong support to the unification-byorientation model of RGs and quasars. For our higher redshift and lower luminosity samples, we find that the ratios of the median lengths in kpc of RGs over quasars for $0<z<1$ is $259 / 157$, for $1<z<2$ is $119 / 86$, for $z>1.5$ is $84 / 77$ and for $z>2$ is actually $56 / 77$. The similarity of the linear size distributions of radio galaxies and quasars can be seen in Fig. 2. We thus conclude that unification without evolution is untenable.

\section{References}

Barthel P.D. (1989) ApJ, 336, 606.

Macklin J.T. (1982) MNRAS, 199, 1119. 